\title{
Upper limits on the mass of supermassive black holes from HST/STIS archival data
}

\author{
E. M. Corsini, ${ }^{1}$ A. Beifiori, ${ }^{1}$ E. Dalla Bontà, ${ }^{1}$ A. Pizzella, ${ }^{1}$ \\ L. Coccato, ${ }^{2}$ M. Sarzi ${ }^{3}$ and F. Bertola ${ }^{1}$ \\ ${ }^{1}$ Dipartimento di Astronomia, Università di Padova, Padova, Italy \\ ${ }^{2}$ Kapteyn Astronomical Institute, University of Groningen, Groningen, The Netherlands \\ ${ }^{3}$ Centre for Astrophysics Research, University of Hertfordshire, Hatfield, UK
}

\begin{abstract}
The growth of supermassive black holes (SBHs) appears to be closely linked with the formation of spheroids. There is a pressing need to acquire better statistics on SBH masses, since the existing samples are preferentially weighted toward early-type galaxies with very massive SBHs. With this motivation we started a project aimed at measuring upper limits on the mass of the SBHs in the center of all the nearby galaxies $(D<100 \mathrm{Mpc})$ for which STIS/G750M spectra are available in the HST archive. These upper limits will be derived by modeling the central emission-line widths observed in the $\mathrm{H} \alpha$ region over an aperture of $\sim 0.1^{\prime \prime}$. Here we present our results for a subsample of $22 \mathrm{~S} 0-\mathrm{Sb}$ galaxies within $20 \mathrm{Mpc}$.
\end{abstract}

Keywords. black hole physics - galaxies: kinematics and dynamics - galaxies: structure

\section{Introduction}

The census of supermassive black holes ( $\mathrm{SBHs}$ ) is large enough to probe the links between mass of SBHs and the global properties of the host galaxies (see Ferrarese \& Ford 2005). However, accurate measurements of SBH masses $\left(M_{\bullet}\right)$ are available for a few tens of galaxies and the addition of new determinations is highly desirable.

To this purpose we started a project aimed at measuring upper limits on $M_{\bullet}$ in the center of all the nearby galaxies $(D<100 \mathrm{Mpc})$ for which STIS/G750M spectra are available in the HST archive. We retrieved data for 213 galaxies spanning over all the morphological types. This will extend previous works by Sarzi et al. (2002) and Verdoes Kleijn et al. (2006). Here we analyze a subsample of 22 galaxies. They have been selected to be S0-Sb within $20 \mathrm{Mpc}$ and to have a measured stellar velocity dispersion $\left(\sigma_{\star}\right)$. All the galaxies were observed as part of the HST/SUNNS project (PI: H.-W. Rix, GO-7361), except for NGC 4435 (Coccato et al. 2006).

\section{Data reduction and analysis}

The STIS/G750M spectra were obtained with the $0.2^{\prime \prime} \times 52^{\prime \prime}$ slit crossing either the galaxy nucleus along a random position angle (SUNNS) or along the galaxy major axis (NGC 4435). The observed spectral region includes the [N II] $\lambda \lambda 6548,6583 \AA$, H $\alpha$, and $[\mathrm{S} \mathrm{II}] \lambda \lambda 6716,6730 \AA$ emission lines. Data reduction was performed with the STIS pipeline, which we implemented to clean cosmic rays and hot pixels. For each galaxy we obtained the nuclear spectrum by extracting a $0.25^{\prime \prime}$-wide $(<25 \mathrm{pc})$ aperture centered on the continuum peak. The ionized-gas velocity dispersion was measured by the fitting Gaussians with the same width and velocity to the narrow component of the [N II] and [S II] emission lines. The $\mathrm{H} \alpha$ line and broad components were fitted with additional Gaussians. 


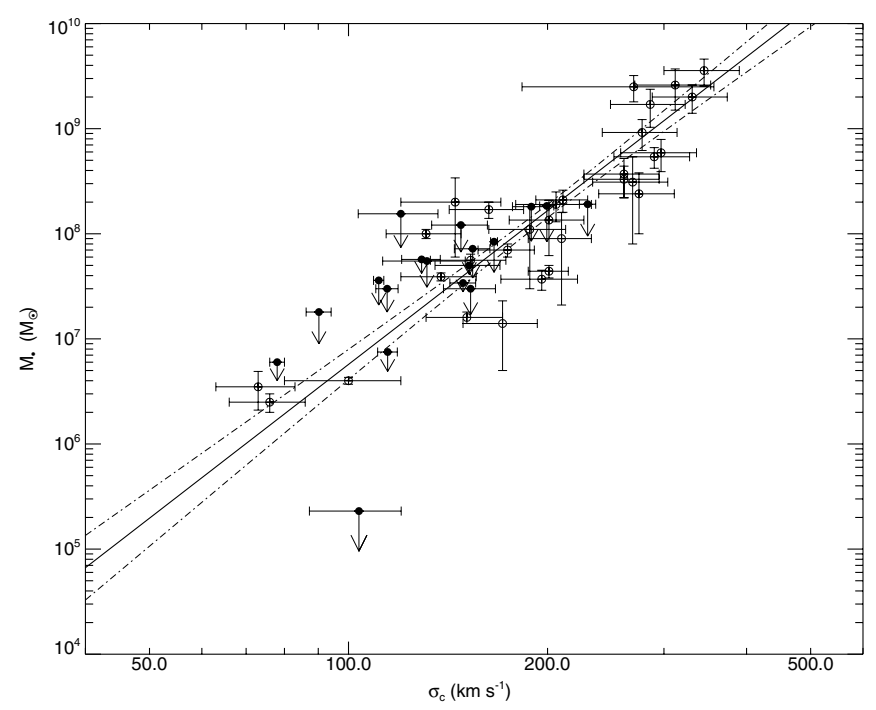

Figure 1. Comparison between our $M_{\bullet}$ upper limits (filled circles) and the $M_{\bullet}-\sigma_{\star}$ relation. The edges of the arrows correspond to upper limits obtained with $i=33^{\circ}$ and $i=81^{\circ}$, respectively. The open circles show the galaxies with accurate measurements of $M \bullet$ (Ferrarese \& Ford 2005).

We assumed that the ionized gas resides in a thin disk and moves onto circular orbits. The local circular velocity is dictated by the gravitational influence of the putative SBH. To derive the upper limits on $M_{\bullet}$ we first built the gaseous velocity field and projected it onto the sky plane according to the disk orientation. Then we observed it by simulating the actual setup of STIS and effects related to the STIS PSF, slit width and the charge bleeding as done by Coccato et al. (2006). Including the mass of the stellar component would lead to tighter upper limits on $M_{\bullet}$. We have no information on the orientation of the gaseous disk within the central aperture. For this pilot project we assumed that the disk is either nearly face on $\left(i=33^{\circ}\right)$ or edge on $\left(i=81^{\circ}\right)$ and the slit is placed along its major axis. We derived the intrinsic flux radial profile of the gaseous disk by fitting a Gaussian function to the narrow emission-line fluxes taking into account of disk inclination and STIS PSF.

\section{Results}

The resulting upper limits are consistent with those derived by Sarzi et al. (2002) for the SUNNS galaxy sample and by Coccato et al. (2006) for NGC 4435. Therefore, we are confident to obtain reliable estimates of the upper limit on $M_{\bullet}$ for all the sample galaxies with emission lines in their spectra. This will allow to increase the statistical significance of the relationships between $M_{\bullet}$ and galaxy properties and identify peculiar objects worthy of further investigations. This is the case of the $M_{\bullet}$ of NGC 3351, NGC 4314, and NGC 4143, which lie below the $M_{\bullet}-\sigma_{\star}$ relation (Fig. 1).

\section{References}

Coccato, L. et al. 2006, MNRAS, 366, 1050

Ferrarese, L. \& Ford, H. 2005, Sp. Sci. Rev., 116, 523

Sarzi, M. et al. 2002, ApJ, 567, 237

Verdoes Kleijn, G. A., van der Marel, R. P. \& Noel-Storr, J. 2006, AJ, 131, 1961 